\title{
Single-Tablet Regimen containing Elvitegravir in an HIV-2 Infected Patient
}

\author{
Ricardo Correia de Abreu*, Duarte F and Neves I
}

Department of Infectious Diseases, Unidade Local de Saúde de Matosinhos, EPE - Hospital Pedro Hispano; Rua Dr. Eduardo Torres, 4454-513 Senhora da Hora, Matosinhos, Portugal

\section{Article Info}

\author{
*Corresponding author: \\ Ricardo Correia de Abreu \\ Rua Dr. Eduardo Torres \\ 4454-513 Senhora da Hora \\ Matosinhos \\ Portugal \\ Tel: +351917018739 \\ E-mail: rca.research@outlook.com
}

Received: January 15, 2019

Accepted: February 27, 2019

Published: March 8, 2019

Citation: Correia de Abreu R, Duarte F, Neves I. Single-Tablet Regimen containing Elvitegravir in an HIV-2 Infected Patient. Madridge J AIDS. 2019; 3(1): 60-61.

doi: $10.18689 / \mathrm{mja}-1000110$

Copyright: (c) 2019 The Author(s). This work is licensed under a Creative Commons Attribution 4.0 International License, which reproduction in any medium, provided the original work is properly cited.

Published by Madridge Publishers permits unrestricted use, distribution, and

\begin{abstract}
Therapeutic armory for HIV-2 infected patients is limited when compared to the one available to HIV-1. Evidence on the use of integrase inhibitors for HIV-2 is still narrow outside clinical trials. The authors present a case report showing the success of a singletabled regimen containing elvitegravir in an HIV-2 infected patient.
\end{abstract}

Keywords: HIV-2; Single-tablet regimen; Elvitegravir/cobicistat; Efficacy; Pregnancy.

\section{Introduction}

HIV-2 was first identified in 1986 in West African patients at Institute Pasteur [1]. Initially typed as non-HIV-1, it shows very similar biological and morphological properties to HIV-1 but presents itself with different antigens and envelope-glycoproteins, making it more closely related to the simian virus. HIV-2 has always been a challenge to treat due to a limited set of drugs and to follow-up, as a result of a lack of correlation between viral load, immunological status and clinical evolution.

The largest HIV-2 cohorts outside Africa are located in the Mediterranean countries like Portugal, Spain, France and Italy even tough with small samples of patients. In the medical literature, real-world examples of the use of integrase inhibitors in HIV-2 patients are scarce and are limited to some clinical trials [2,3]. Most studies show clear efficacy of raltegravir in these patients, but the examples described with the use of elvitegravir or dolutegravir are still rare, both of which offer the possibility of singletablet regimens [3-6].

\section{Case Report}

The authors describe the case of a 22-year-old woman from Guinea-Bissau living in Portugal since June 2014. She was referred to the Infectious Diseases Department in August of the same year after the diagnosis of HIV infection in the context of a pregnancy that was then voluntarily discontinued. Blood tests showed CD4+ T-cell count of 109/ $\mathrm{mL}$, positive Western Blot for HIV-1 and 2, HIV-2 RNA of 2780 copies/mL, (RT-PCR testnegative for HIV-1). Other blood tests showed a normal blood count, renal and liver function, slight increase in total cholesterol $(205 \mathrm{mg} / \mathrm{dL})$, remaining negative viral serologies as well as VDRL and TPHA.

On October 2014 she was started on tenofovir disoproxil fumarate/emtricitabine (300/200 mg once daily) plus raltegravir ( $400 \mathrm{mg}$ twice daily) and trimetoprim/sulfametoxazol (960 mg every alternate day) for opportunistic infections (Ols) prophylaxis. Clinically she showed a gradual rise in CD4+ T cells and a sustained virological suppression over time.

In September 2015 the patient showed some compliance issues focusing on the prescription schedule due to a delicate family situation. For fear of non-compliance with 
consequent immune repercussion, viral rebound and/or acquisition of viral resistance, she was switched to a single-tablet regimen with tenofovir disoproxil fumarate/emtricitabine/ elvitegravir/cobicistat (300/200/150/150 mg once daily). Since then, the patient showed a good compliance, a sustained immunological recovery (being able to stop Ols prophylaxis) and persistent virological suppression.

In September 2017 the patient became pregnant and during this period the therapeutic regimen was maintained without virological failures or side effects. Delivery was by cesarean section without intercurrences and HIV-2 infection in the child was excluded after regular monitoring as suggested by the international guidelines.

In November 2018 the patient was switched to tenofovir alafenamide/emtricitabine/elvitegravir/cobicistat (10/200/150/150 mg once daily) maintaining a good compliance, sustained immunological recovery and virological suppression.

\section{Discussion and Conclusion}

This case allows us to explore an important issue in pharmacological armory approved so far for HIV-2 infected patients. Data on the use of integrase inhibitors for HIV-2 infection is limited and little ongoing research on new drugs for HIV-2 exist [2,3]. The existence of single tablet regimen including an integrase inhibitor with demonstrated efficacy in HIV-2, may be another useful weapon to help overcome issues of poor adherence, reducing the risk of virological failure and acquisition of resistance $[5,6]$. Although this is a unique case, it allows us to verify that the option of using elvitegravir in a single-tablet regimen in HIV-2 patients, even during pregnancy, is effective in immunological recovery and viral suppression, as well as in the absence of effects adverse effects.

\section{Conflicts of Interest}

None of the authors have conflicts of interest.

\section{References}

1. Clavel $F$, Mansinho $K$, Chamaret $S$, et al. Human immunodeficiency virus type 2 infection associated with AIDS in West Africa. N Engl J Med. 1987; 316(19): 1180-1185.

2. Gottlieb GS, Smith RA, Dia Badiane NM, et al. HIV-2 Integrase Variation in Integrase Inhibitor-Naïve Adults in Senegal, West Africa. PLoS One. 2011; 6(7): e22204. doi: 10.1371/journal.pone.0022204

3. Ba S, Raugi DN, Smith RA, et al. A Trial of a Single-tablet Regimen of Elvitegravir, Cobicistat, Emtricitabine, and Tenofovir Disoproxil Fumarate for the Initial Treatment of Human Immunodeficiency Virus Type 2 Infection in a Resource-limited Setting: 48-Week Results From Senegal West Africa. Clin Infect Dis. 2018; 67(10): 1588-1594. doi: 10.1093/cid/ ciy324

4. Zheng $Y$, Lambert $C$, Arendt $V$, Seguin-Devaux C. Virological and immunological outcomes of elvitegravir-based regimen in a treatmentnaïve HIV-2-infected patient. AIDS. 2014; 28(15): 2329-2331. doi: 10.1097/ QAD.00000000000000414

5. Descamps D, Peytavin G, Visseaux B, et al. Dolutegravir in HIV-2-Infected Patients with Resistant Virus to First-line Integrase Inhibitors from the French Named Patient Program. Clin Infect Dis. 60(10): 1521-1527. doi: 10.1093/cid/civ124

6. Treviño $A$, Cabezas $T$, Lozano $A B$, et al. Dolutegravir for the treatment of HIV-2 infection. J Clin Virol. 64: 12-15. doi: 10.1016/j.jcv.2015.01.001 